\title{
Distinguishing Cartesian Powers of Graphs
}

\author{
Michael O. Albertson \\ Department of Mathematics \\ Smith College, Northampton, MA 01063 USA \\ albertson@math.smith.edu
}

Submitted: Feb 26, 2005; Accepted: Sep 1, 2005; Published: Sep 19, 2005

Mathematics Subject Classifications: 05C25, 05C78

\begin{abstract}
Given a graph $G$, a labeling $c: V(G) \rightarrow\{1,2, \ldots, d\}$ is said to be $d$-distinguishing if the only element in $\operatorname{Aut}(G)$ that preserves the labels is the identity. The distinguishing number of $G$, denoted by $D(G)$, is the minimum $d$ such that $G$ has a $d$-distinguishing labeling. If $G \square H$ denotes the Cartesian product of $G$ and $H$, let $G^{2}=G \square G$ and $G^{r}=G \square G^{r-1}$. A graph $G$ is said to be prime with respect to the Cartesian product if whenever $G \cong G_{1} \square G_{2}$, then either $G_{1}$ or $G_{2}$ is a singleton vertex. This paper proves that if $G$ is a connected, prime graph, then $D\left(G^{r}\right)=2$ whenever $r \geq 4$.
\end{abstract}

\section{Introduction}

Given a graph $G$, a labeling $c: V(G) \rightarrow\{1,2, \ldots, d\}$ is $d$-distinguishing if the only element in $\operatorname{Aut}(G)$ that preserves the labels is the identity. The idea is that the labeling together with the structure of $G$ uniquely identifies every vertex. Formally, $c$ is said to be $d$-distinguishing if $\phi \in \operatorname{Aut}(G)$ and $c(\phi(x))=c(x)$ for all $x \in V(G)$ implies that $\phi=i d$. The distinguishing number of $G$, denoted by $D(G)$, is the minimum $d$ such that $G$ has a $d$-distinguishing labeling. It is a measure of the relative symmetry of $G$.

It is immediate that $D\left(K_{n}\right)=n$ and when $q>p, D\left(K_{p, q}\right)=q$. It is straightforward to see that $D\left(K_{n, n}\right)=n+1$. The original paper on distinguishing [1] was inspired by a recreational puzzle [5]. The solution requires showing that if $n \geq 6$, then $D\left(C_{n}\right)=2$. The attraction of this puzzle is the contrast with smaller cycles where $D\left(C_{n}\right)=3$ when $3 \leq n \leq 5$.

The inspiration for this paper is the solution to the problem of distinguishing the generalized cubes. Let $Q_{r}$ denote the $r$-dimensional hypercube: $V\left(Q_{r}\right)=\left\{\mathbf{x}=\left(x_{1}, \ldots, x_{r}\right)\right.$ : $\left.x_{i} \in \mathbb{Z}_{2}\right\}$ and $\mathbf{x y} \in E\left(Q_{r}\right)$ if $\mathbf{x}$ and $\mathbf{y}$ differ in exactly one coordinate. Note that $Q_{2}=C_{4}$, $Q_{3}$ is the standard cube, and $D\left(Q_{2}\right)=D\left(Q_{3}\right)=3$. 
The Cartesian (or box) product of two graphs $G$ and $H$, denoted by $G \square H$, is the graph whose vertex set $V(G \square H)=\{(u, v): u \in V(G), v \in V(H)\}$. The vertex $(u, v)$ is adjacent to the vertex $(w, z)$ if either $u=w$ and $v z \in E(H)$ or $v=z$ and $u w \in E(G)$. The box notation illustrates the Cartesian product of two edges. Here we let $G^{2}$ denote $G \square G$ and recursively let $G^{r}=G \square G^{r-1}$. The connection between hypercubes and Cartesian products is that $Q_{r}=K_{2}^{r}$. For more on Cartesian products see [4].

Recently Bogstead and Cowen showed that if $r \geq 4$, then $D\left(Q_{r}\right)=2$ [2]. Their proof idea is elegant: find $H$, an induced subgraph of $G$, such that any nontrivial automorphism of $G$ maps some vertex in $H$ to a vertex not in $H$. In such a circumstance the natural labeling $\{c(x)=2$ if $x \in V(H)$ and $c(x)=1$ otherwise $\}$ is 2-distinguishing. Using this technique it is straightforward to prove that $D\left(K_{3}^{3}\right)=D\left(P_{3}^{2}\right)=2$, and it is natural to think that larger powers of these graphs will also be 2-distinguishable. All of this suggests the following conjecture.

Conjecture 1. If $G$ is connected, then there exists $R=R(G)$ such that if $r \geq R$, then $D\left(G^{r}\right)=2$.

The connectivity is necessary since if $G$ is two independent vertices, then $D\left(G^{r}\right)=2^{r}$.

This purpose of this note is to prove Theorem 2, a significant strengthening of the above conjecture for a slightly smaller class of graphs. In its full generality Conjecture 1 remains open.

\section{Cartesian Products}

A graph $H$ is said to be prime with respect to the Cartesian product if whenever $H \cong$ $H_{1} \square H_{2}$, then either $H_{1}$ or $H_{2}$ is a singleton vertex. It is well known that if $G$ is connected, then $G$ has a unique prime factorization i.e. $G \cong H_{1} \square H_{2} \square \cdots \square H_{t}$ such that for $1 \leq i \leq$ $t, H_{i}$ is prime. About thirty-five years ago Imrich and Miller independently showed the following theorem.

Theorem 1. [4] If $G$ is connected and $G=H_{1} \square H_{2} \square \cdots \square H_{r}$ is its prime decomposition, then every automorphism of $G$ is generated by the automorphisms of the factors and the transpositions of isomorphic factors.

Corollary 1.1. If $G$ is a connected prime graph with $|V(G)|=n$, then $\operatorname{Aut}\left(G^{r}\right) \leq$ $\operatorname{Aut}\left(K_{n}^{r}\right)$

Proof. Since every automorphism of $G$ is an automorphism of $K_{n}$, it follows that every automorphism of $G^{r}$ is an automorphism of $K_{n}^{r}$.

Corollary 1.2. If $G$ is a connected prime graph with $|V(G)|=n$, then $D\left(G^{r}\right) \leq D\left(K_{n}^{r}\right)$.

Proof. Any labeling that destroys every automorphism of $K_{n}^{r}$ must also destroy every automorphism of $G^{r}$. 
We now state our main result, though its proof will be postponed until the end of the next section.

Theorem 2. If $G$ is a connected graph that is prime with respect to the Cartesian product, then $D\left(G^{r}\right)=2$ whenever $r \geq 4$. Futhermore, if in addition, $|V(G)| \geq 5$, then $D\left(G^{r}\right)=2$ whenever $r \geq 3$.

It is well known that almost all graphs are connected. Graham [3] has shown that almost all graphs are irreducible with respect to the $\Theta^{*}$ equivalence class; see [4]. Since every such irreducible graph is prime, almost all graphs satisfy the hypotheses of Theorem 2 .

It seems that it should be possible to prove Theorem 2 using the Bogstead Cowen strategy. Whether there is such a proof remains open.

\section{The Motion Lemma and Its Consequences}

For $\sigma \in \operatorname{Aut}(G)$ let $m(\sigma)=|\{x \in V(G): \sigma(x) \neq x\}|$ and let $m(G)=\min \{m(\sigma)$ : $\sigma \neq i d\}$. Call $m(\sigma)$ the motion of $\sigma$ and $m(G)$ the motion of $G$. Using an appealing probabilistic argument Russell and Sundaram showed that if the motion of $G$ is large, then the distinguishing number of $G$ is small. Specifically they proved the motion lemma, Theorem 3.

Theorem 3. [6] If $d^{\frac{m(G)}{2}}>|\operatorname{Aut}(G)|$, then $D(G) \leq d$.

To apply the motion lemma we need determine $\left|\operatorname{Aut}\left(K_{n}^{r}\right)\right|$ and $m\left(K_{n}^{r}\right)$.

Theorem 4. $\left|\operatorname{Aut}\left(K_{n}^{r}\right)\right|=r !(n !)^{r}$.

Proof. $K_{n}^{r}$ is vertex transitive and has $n^{r}$ vertices. Each vertex, say $x$, is contained in exactly $r$ cliques of size $n$ and the vertices in these cliques are disjoint except for $x$. An automorphism might take $x$ to any of the $n^{r}$ vertices. Once the image of $x$ is chosen, then a clique that contains $x$ can be mapped to a clique that contains the image of $x$ in any of $r(n-1)$ ! ways. A second clique containing $x$ can be mapped in any of $(r-1)(n-1)$ ! ways. The $j^{\text {th }}$ clique containing $x$ can be mapped in any of $(r-j+1)(n-1)$ ! ways. Once all cliques containing $x$ are mapped, the entire automorphism is fixed. Alternatively, one can recognize $\operatorname{Aut}\left(K_{n}^{r}\right)$ as an appropriate wreath product and arrive at the count that way.

Theorem 5. If $n \geq 3$, then $m\left(K_{n}^{r}\right)=2 n^{r-1}$.

Proof. For every $x_{2}, \ldots, x_{r}$, let $\sigma_{0}$ be the automorphism of $K_{n}^{r}$ in which $\sigma_{0}\left(1, x_{2}, \ldots, x_{r}\right)=$ $\left(2, x_{2}, \ldots, x_{r}\right) ; \sigma_{0}\left(2, x_{2}, \ldots, x_{r}\right)=\left(1, x_{2}, \ldots, x_{r}\right)$; and $\sigma_{0}$ fixes everything else. Clearly $m\left(\sigma_{0}\right)=2 n^{r-1}$. It remains to show that no non-trivial automorphism has smaller motion.

The proof that $m\left(K_{n}^{r}\right) \geq 2 n^{r-1}$ will use a combination of induction and contradiction. The base case holds since when $r=1$, any non-identity automorphism must move at least two vertices. 
Let $F_{j_{1}, j_{2}, \ldots, j_{t}}=\left\{\left(x_{1}, \ldots, x_{r}\right) \in V\left(K_{n}^{r}\right): x_{1}=j_{1}, x_{2}=j_{2}, \ldots, x_{t}=j_{t}\right\}$. The notation is chosen to emphasize that we are looking at vertices in $K_{n}^{r}$ whose first coordinates are fixed. Let $L_{k}=\left\{\left(x_{1}, \ldots x_{r}\right) \in V\left(K_{n}^{r}\right): x_{r}=k\right\}$. The notation is chosen to emphasize that we are looking at vertices in $K_{n}^{r}$ whose last coordinate is fixed. Note that $\left|F_{j_{1}, j_{2}, \ldots, j_{t}}\right|=n^{r-t}$ and that $\left|L_{k}\right|=n^{r-1}$.

If $\sigma \in \operatorname{Aut}\left(K_{n}^{r}\right)$ is such that $0<m(\sigma)<2 n^{r-1}$, then $\sigma$ fixes more than $(n-2) n^{r-1}$ vertices. By the pigeonhole principle and appropriate reindexing there exists $j_{1}, j_{2}, \ldots, j_{r-1}$ such that $\sigma$ fixes more than $(n-2) n^{r-2}$ vertices in $F_{j_{1}} ; \sigma$ fixes more than $(n-2) n^{r-3}$ vertices in $F_{j_{1}, j_{2}} ; \sigma$ fixes more than $(n-2) n^{r-s-1}$ vertices in $F_{j_{1}, j_{2}, \ldots, j_{s}}$; and $\sigma$ fixes more than $n-2$ vertices in $F_{j_{1}, \ldots, j_{r-1}}$. Alternatively $\sigma$ moves at most one vertex in this clique. Since $n \geq 3, \sigma$ fixes the entire clique $F_{j_{1}, \ldots, j_{r-1}}$.

For $1 \leq k \leq n, L_{k} \cap F_{j_{1}, \ldots, j_{r-1}}=\left\{\left(j_{1}, j_{2}, \ldots, j_{r-1}, k\right)\right\}$. This vertex is fixed by $\sigma$. Now any vertex in $K_{n}^{r}$ that is adjacent to $\left(j_{1}, j_{2}, \ldots, j_{r-1}, k\right)$ is either in $F_{j_{1}, \ldots, j_{r-1}}$ or in $L_{k}$. In the former case it is fixed by $\sigma$. In the latter case in order to preserve adjacency, it must be mapped to a vertex in $L_{k}$. Now all the vertices in $L_{k}$ that are at distance two from $\left(j_{1}, j_{2}, \ldots j_{r-1} k\right.$ must also be mapped to $L_{k}$. Continuing we see that $\sigma$ maps $L_{k}$ to itself.

Next, for the moment suppose that for a particular value of $k, L_{k}$ is fixed by $\sigma$. Since every vertex in $K_{n}^{r}-L_{k}$ is adjacent to exactly one vertex in $L_{k}, \sigma$ must map $L_{1}, L_{2}, \ldots L_{n}$ onto $L_{1}, L_{2}, \ldots, L_{n}$. Since $\sigma$ is the identity on $F_{j_{1}, \ldots, j_{r-1}}, \sigma$ is the identity on all of $K_{n}^{r}$.

Thus we may assume that for every $k$ with $1 \leq k \leq n, \sigma$ maps $L_{k}$ to $L_{k}$ moving some of the vertices in $L_{k}$. Since $\left.\sigma\right|_{L_{k}}$ is an automorphism on $K_{n}^{r-1}$ we can inductively assume that $\sigma$ moves at least $2 n^{r-2}$ vertices. Since this is true for each $k, m(\sigma) \geq 2 n^{r-1}$.

We now turn to the proof of Theorem 2 .

Proof. First we note that when $r>1, G^{r}$ is not rigid. Thus $D\left(K_{n}^{r}\right)>1$. If $n=2$, then Theorem 2 is just the result of Bogstead and Cowen. When $n \geq 3$ we can substitute the results of Theorems 3 and 4 into the Motion Lemma. Thus if $r !(n !)^{r}<2^{n^{(r-1)}}$, then $D\left(K_{n}^{r}\right) \leq 2$.

Case (i): Suppose $n \geq r \geq 4$. It is straightforward to check the following inequalities. The logarithms are base 2 .

$$
\log (r !)+r \log (n !)<n \log (n)+n^{2} \log (n)<n^{3} \leq n^{r-1} .
$$

Exponentiating the extremes gives $r !(n !)^{r}<2^{n^{(r-1)}}$.

Case (ii): Suppose $r>n \geq 3$ and $r \geq 5$. It is straightforward to check the following inequalities. The logarithms are base 2 .

$$
\log (r !)+r \log (n !)<r \log (r)+r^{2} \log (r)<3^{r-1} \leq n^{r-1} .
$$

Again exponentiating the extremes gives $r !(n !)^{r}<2^{n^{(r-1)}}$.

Case (iii): Suppose $r=4$ and $n=3$. A direct calculation shows that $r !(n !)^{r}<2^{n^{(r-1)}}$.

Finally it is straightforward to check that if $r=3$ and $n \geq 5,6(n !)^{3}<2^{n^{2}}$. 
Acknowledgments: Thanks to Tom Tucker and Mark Watkins for helpful discussions. This research began while the author was the Neil R. Grabois Visiting Chair in Mathematics at Colgate University.

\section{References}

[1] Michael O. Albertson and Karen L. Collins, Symmetry breaking in graphs. Electronic J. Combinatorics 3 (1996) \#R18.

[2] Bill Bogstad and Lenore J. Cowen The distinguishing number of the hypercube. Discrete Math. 283 (2004), no. 1-3, 29-35.

[3] Ronald Graham, Isometric embeddings of graphs, in Selected Topics in Graph Theory 3, Academic Press, San Diego CA, 1988, 133-150.

[4] Wilfried Imrich and Sandi Klavžar, Product Graphs, John Wiley \& Sons, Inc. New York, 2000.

[5] Frank Rubin, Problem 729: the blind man's keys, Journal of Recreational Mathematics, 11(2) (1979), 128, solution in 12(2) (1980).

[6] Alexander Russell and Ravi Sundaram, A note on the asymptotics and computational complexity of graph distinguishability. Electronic Journal of Combinatorics, 5 (1998) \#R2. 